\title{
Indirect Genetic Effects for Growth in Pigs Affect Behaviour and Weight Around Weaning
}

\author{
Irene Camerlink $^{1,2,4} \oplus \cdot$ Winanda W. Ursinus $^{1,3} \cdot$ Andrea C. Bartels $^{1,2} \cdot$ Piter Bijma $^{2} \cdot$ J. Elizabeth Bolhuis ${ }^{1}$
}

Received: 25 March 2018 / Accepted: 11 June 2018 / Published online: 19 June 2018

(c) The Author(s) 2018

\begin{abstract}
Selection for indirect genetic effects (IGE), i.e. the genetic effect of an individual on a trait of another individual, is a promising avenue to increase trait values in plant and animal breeding. Studies in livestock suggest that selection for IGE for growth (IGEg) might increase animals' capacity to tolerate stress. We assessed the effect of a stressful phase (weaning) on the behaviour and performance of pigs $(n=480)$ divergently selected for high or low IGEg. High IGEg pigs were significantly slower to explore the feed and gained less weight than low IGEg pigs in the days after weaning. In line with previous findings, high IGEg animals may have prioritized the formation of social ranks.
\end{abstract}

Keywords Pig $\cdot$ Weaning $\cdot$ Indirect genetic effects $\cdot$ Breeding $\cdot$ Genotype-by-environment $\cdot$ Enrichment

\section{Introduction}

Group living individuals inevitably affect each other through their social interactions. Social interactions are partly genetic, and this genetic component is known as an indirect genetic effect (IGE). IGEs, also termed associative effects or social genetic effects, are the genetic effects of an individual on another conspecific (Griffing 1967; Moore et al. 1997; Wolf et al. 1998; Muir 2005; Bijma et al. 2007). IGEs have become an established concept in behavioural ecology (Bailey et al. 2017), livestock breeding (Ellen et al. 2014)

Edited by Stephen Maxson.

Winanda W. Ursinus-Part of this research is undertaken in a personal capacity, Lienden, The Netherlands.

Irene Camerlink

Irene.camerlink@vetmeduni.ac.at

1 Adaptation Physiology Group, Wageningen University and Research, Wageningen, The Netherlands

2 Animal Breeding and Genomics, Wageningen University and Research, Wageningen, The Netherlands

3 Animal Behaviour \& Welfare, Wageningen Livestock Research, Wageningen University and Research, Wageningen, The Netherlands

4 Institute of Animal Husbandry and Animale Welfare, University for Veterinary Medicine Vienna, Veterinärplatz 1, 1210 Vienna, Austria and plant breeding (Bijma 2014). IGE models capture the genetic effect of an individual on the performance of another individual in the same group (in case of animals) or vicinity (in case of trees). Thereby IGEs for performance traits contribute to the accuracy of the breeding value predictions and can increase the progress in the population's performance without the need for additional phenotyping beyond the main trait of interest (Bijma et al. 2007; Bijma and Wade 2008). Hence, incorporating IGEs in the breeding strategies has attracted much interest in agriculture (Ellen et al. 2014).

IGEs do not relate to one specific social interaction, but instead capture the overall effect of several social interactions between individuals on a specific trait of the recipient individual. This can relate to any form of interaction between individual organisms, including social behaviour in animals (Ellen et al. 2014), competition for light, water and nutrients between plants (e.g. Muir 2005; Costa e Silva and Kerr 2013) or transmission of disease (animals: LipschutzPowell et al. 2012; forest tree: Potts et al. 2013).

Selection on IGEs is always for a certain trait, for example the IGE for survival (e.g. Peeters et al. 2012), pheromones (Duijvesteijn et al. 2012), or for growth performance (Bergsma et al. 2013). The IGE for growth, here abbreviated as IGEg, is the genetic effect of an individual on the growth performance of its group mates. In a one-generation selection experiment in which pigs where divergently selected for IGEg, animals selected for high IGEg showed behavioural changes related to a reduction 
in aggressive and non-aggressive biting behaviour (Camerlink et al. 2015). They appeared to be less fearful in behavioural tests (Reimert et al. 2014a) and their immune status suggested a lower stress susceptibility (Reimert et al. 2014b). Because of the range of behavioural and physiological changes related to the attempt to cope with stress, it has been suggested (in Camerlink 2014) that selection on IGEg might alter stress sensitivity. Stress sensitivity may, therefore, be an underlying mechanism explaining the difference in social behaviour between pigs with diverging IGEg. We here address the relationship between IGEg and stress sensitivity, which has to our knowledge not been studied previously.

In current pig husbandry, pigs face many stressful events such as handling by humans, weaning from the sow, relocation to an unknown environment and mixing with unfamiliar conspecifics (Martínez-Miró et al. 2016). Here, individual differences exist in stress sensitivity (e.g. Bolhuis et al. 2004; Ursinus et al. 2013). Pigs are particularly challenged when weaned under commercial husbandry conditions (Campbell et al. 2013). Weaning includes the separation of the young from the mother and in pig husbandry this often coincides with rough handling by stock workers, transport, a new environment, novel feed, and the first encounter with unfamiliar conspecifics. As a consequence, piglets often show a behavioural and physiological stress response in the first days after weaning (Oostindjer et al. 2011a). Typically, piglets show a reluctance to eat, a decrease in growth or even a loss in body weight and restlessness in the early post-weaning period. Housing conditions may alter the response to weaning, with in general a better adaptation to the new situation when enrichment material that encourages exploration, such as straw, is provided (Melotti et al. 2011; Oostindjer et al. 2011b, c). Exploration will aid in getting the animals familiarized with the new surroundings, including where to find feed and water, and can therefore reduce stress and increase feed intake (Oostindjer et al. 2011c).

We hypothesized that animals selected for positive IGEg (higher IGEg than population average)-i.e. estimated to have a positive influence on the growth of their group mates-would cope better with the stressful conditions surrounding weaning as compared to pigs with a negative IGEg (IGEg below population average)-i.e. estimated to have a negative effect on the growth of their group members. We expect an additive effect of the housing conditions, with a better coping response in animals housed in straw enriched conditions. Here we studied the ability of pigs to cope with the stressful conditions surrounding weaning, measured by their behaviour and weight, using 480 pigs that were divergently selected for IGEg and housed in either conventional barren pens or straw enriched pens after weaning.

\section{Methods}

The experimental protocol was approved by the Institutional Animal Care and Use Committee of Wageningen University (Protocol Number: 2010055f), the Netherlands. The protocol was in accordance with recommendations of the European Guidelines for accommodation and care of animals.

\section{Animals and housing}

The animals in this study were the same as in Camerlink et al. (2013) and Ursinus et al. (2014a). Eighty sows (Topigs-20) were serviced by Tempo boars to create a contrast (high vs. low) in estimated Indirect Genetic Effects for growth (IGEg). Detailed information on the genetic estimates can be found in Camerlink et al. (2013, 2014). In short, high IGEg offspring (40 litters) were expected to increase the growth of their pen mates, whereas low IGEg offspring (40 litters) were expected to decrease the growth of their pen mates. Dams and sires with the most extreme high and low IGEg of the available population were selected, and their offspring were studied. The contrast between high and low IGEg offspring was $14 \mathrm{~g} /$ day average daily gain (ADG) (based on six pigs per pen, $(6-1) \times 2.8 \mathrm{~g} /$ day $=14 \mathrm{~g} /$ day; Camerlink et al. 2015). Offspring ( $n=1210)$ were born over five consecutive batches (i.e. farrowing groups).

Sows and piglets were kept at the experimental farm of TOPIGS Research Center IPG in Beilen, the Netherlands. A detailed description about housing and management can be found in Camerlink et al. (2013) and Ursinus et al. (2014a). Briefly, piglets were kept in barren conventional farrowing crates $\left(3.8 \mathrm{~m}^{2}\right.$ with $53 \%$ slatted flooring). If the number of piglets in a litter exceeded 14 then fostering was applied within the same treatment group. Males were castrated before 5 days of age for production purposes. Water was available ad libitum via a drinking nipple and after one week piglets had access to a commercial diet.

Piglets were weaned at four weeks of age. At weaning all piglets were weighed and assessed for health. A total of 480 piglets were selected for follow up based on absence of clear disease symptoms, sex (1:1 ratio), and backtest response (see below). Selected piglets $(n=480)$ received an ear tag with identification number and were then transported over a $2.5 \mathrm{~h}$ truck journey to experimental facilities "De Haar", Wageningen, the Netherlands. During transport, contact between unfamiliar conspecifics was prevented by keeping only siblings together in isolated groups. Upon arrival the piglets were grouped-within the same IGEg group - so that each group consisted of six 
unacquainted piglets in either a barren or an enriched pen. Both housing types measured $6.7 \mathrm{~m}^{2}$. The barren pens (B) had a $47 \%$ slatted floor and a chain with ball hanging from the wall. The enriched pens $(\mathbf{E})$ had a solid floor covered with a deep litter layer of straw and wood shavings, and a chain with ball hanging from the wall. Feed was offered ad libitum in a feeder with three feeding places. Water was available via a drinking nipple. Per batch, 16 pens of six piglets each were studied, resulting in a total of 80 pens in a $2 \times 2$ experimental arrangement with IGEg (high vs. low) and housing (barren vs. enriched) as treatments.

\section{Backtest response pre-weaning}

At $~ 14$ days of age, piglets were subjected to a backtest following the methods of Hessing et al. (1993) and Bolhuis et al. (2003). In the backtest a piglet is placed in a supine position for one minute, during which the piglet's response is monitored. The number of struggles, also called escape attempts, together with the number of vocalizations, can give an indication of a piglet's coping style. Hereby, piglets are commonly classified into either high resisting (HR) or low resisting (LR), based on the distribution of the continuous scale (Iversen et al. 2017). Previously, no relationship between backtest classification and IGEg was found (Reimert et al. 2013). The backtest classification was here predominantly used to standardize group composition, as the behaviour of animals with different coping styles may profoundly differ. Group composition post-weaning was standardized with a LR:HR ratio in accordance with the whole tested population; meaning that each pen contained at least two pigs of each backtest classification and at least one HR male and one HR female (Ursinus et al. 2014a; Camerlink et al. 2013).

\section{Body weight}

Weight gain of piglets in the first 4 days after weaning was used as indicator of an individual's ability to cope with the stressful conditions surrounding weaning. Body weight of piglets was recorded at weaning (before transport to the experimental facilities; 27 days of age) and 4 days after. Piglets were weighed individually by placing them in a crate onto a weigh scale appropriate for the size of the piglets at that age. Average daily gain (ADG) in g per day (g/d), was calculated by dividing the weight gain by the 4 days.

\section{Behavioural observations}

Behaviour, as an indicator of coping ability, was recorded through video observations. Video cameras were mounted to the ceiling to record the pens in top view with a small angle. Each camera captured two pens at the same time in full colour at 25 frames per sec. Video data were recorded with Geovision during the day of weaning and the day after. For recognition during behavioural observations, piglets received a number sprayed on their back with a stock marker upon entering the pen at weaning. Behaviour was then observed from video by three trained observers. For each pig they recorded the latency (in min.) to explore the feed / eat, to lie down individually and to lie down together with at least two other pigs. See Table 1 for the definitions used for scoring these behaviours. Lying down together with at least two others was chosen so that it would be more than random contact of, for example, the hind legs touching each other. Latencies were recorded from the moment the individual piglet was placed in the pen up to maximum $5 \mathrm{~h}$ after. If an animal did not show one of the scored behaviours within $5 \mathrm{~h}$ then the latency was set to $5 \mathrm{~h}(300 \mathrm{~min})$.

\section{Data analysis}

Data were analysed with SAS version 9.3. The response variables were weight, ADG, latency to explore feed, latency to lie down, and latency to lie down in a group. From the 480 pigs, 466 were included in the analysis for the latency to explore feed and to lie down individually, whereas the latency to lie down together was recorded for 165 pigs (two batches only). The residuals of weight, ADG, and the latencies to lie down individually and in a group were normally distributed but the residuals of the latency to eat were skewed and had to be log transformed before reaching a normal distribution.

Data were analysed in Generalized Linear Models (PROC MIXED) to include the random effects for batch, group of pigs (pen) and sow (i.e. dam). The fixed effects in each model where the genetic line (i.e., High or Low IGEg) and housing conditions (B / E), their interaction (testing Genotype $\times$ Environment, i.e. $\mathrm{G} \times \mathrm{E}$ effects), sex $(\mathrm{M} / \mathrm{F})$ and coping style (HR / LR). Values are Least Square Means with SEM unless stated otherwise (e.g. back-transformed means).
Table 1 Ethogram for behavioural observations from video

\begin{tabular}{lll}
\hline Behaviour & Description & Observation \\
\hline Exploring/eating feed & Head is in the feed trough $\geq 3 \mathrm{~s}$ continuously & By individual \\
Lying down individually & Belly touches the floor for $\geq 5 \mathrm{~s}$ & By individual \\
Lying down together & Lying in body contact with at least two other pigs & By individual \\
\hline
\end{tabular}




\section{Results}

\section{Weight gain after weaning}

At weaning the weight of High and Low IGEg pigs did not differ significantly $\left(F_{1,335}=2.14 ; P=0.14\right)$ (Camerlink et al. 2014). Four days later, piglets of the High IGEg group were lighter than piglets from the Low IGEg group (High $8.93 \pm 0.18 \mathrm{~kg}$; Low $9.30 \pm 0.19 \mathrm{~kg} ; F_{1,335}=5.08$; $P=0.02$; Fig. 1). The weight difference was also reflected in a lower ADG for High IGEg pigs as compared to Low IGEg pigs in the first 4 days after weaning (High $160 \pm 2 \mathrm{~g}$ ADG; Low $200 \pm 2$ g ADG; $\left.F_{1,335}=5.69 ; P=0.02\right)$. There was no overall effect of housing conditions, sex or coping style on the weight or ADG at weaning or in the first 4 days after weaning (all $P>0.10$ ). There were no significant $\mathrm{G} \times \mathrm{E}$ interactions for weight or ADG.

\section{Behaviour at weaning}

On average, pigs started investigating the feed at $17 \mathrm{~min}$ $(995 \pm 65 \mathrm{~s}$; raw means) after arriving into the new facilities (head in the feeder for at least $3 \mathrm{~s}$ ). This latency to explore or eat the feed was significantly affected by the genetics. High IGEg pigs took double the time compared to Low IGEg pigs before they reached for the feeder High $6.48 \pm 0.3 \log , 651 \mathrm{~s}, 11 \mathrm{~min} ;$ Low $5.83 \pm 0.3 \log , 339 \mathrm{~s}$, 6 min; $F_{1,321}=17.60 ; P<0.001 ;$ Fig. 2 . There were no effects of housing conditions (Fig. 2), sex or coping style, and there was no $\mathrm{G} \times \mathrm{E}$ interaction for the latency to explore feed.

It took on average $56 \pm 2 \mathrm{~min}$ before pigs were lying down individually. The genetics did not influence the time to lie down $\left(F_{1,264}=0.48 ; P=0.49\right)$, but pigs in the barren

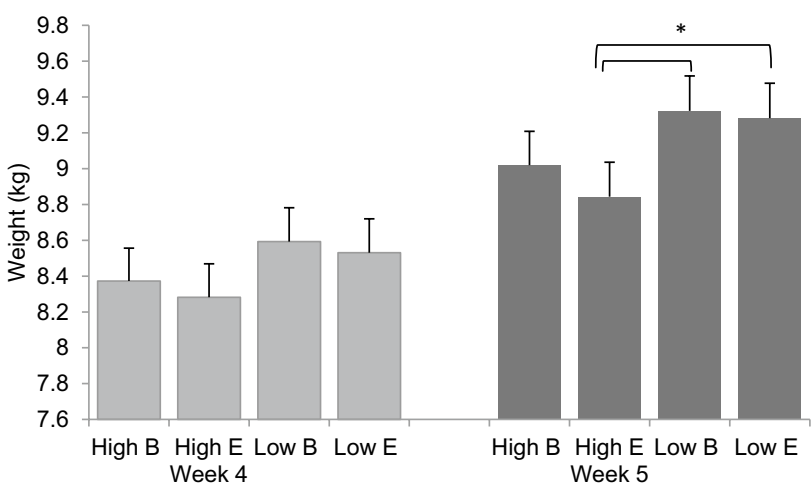

Fig. 1 Means with SE for body weight in kg, by IGEg group (High/ Low) and housing condition (Barren, B/Enriched, E), at weaning (week 4) and 4 days later (week 5)

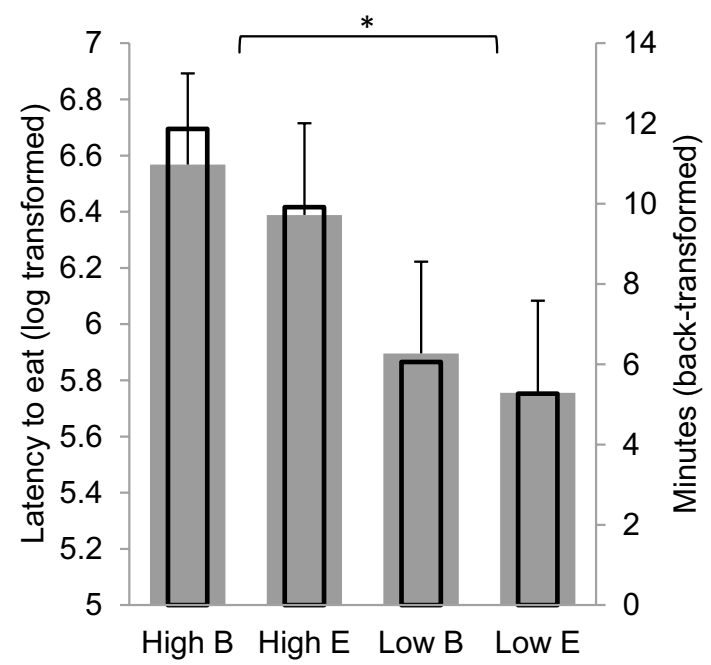

Fig. 2 Mean \pm SE latency on log transformed scale (grey bars) and back-transformed to minutes (thinner transparent bars) to start exploring or eating the feed after arrival in the experimental facilities (after being weaned and transported), by IGEg group (High / Low) and housing condition (Barren, B / Enriched, E)

conditions lay down sooner than pigs in straw enriched pens $\left(F_{1,264}=9.79 ; P<0.01 ;\right.$ Fig. 3$)$. Sex and coping style did not influence the latency to lie down individually.

After on average $108 \pm 3 \mathrm{~min}(13 / 4 \mathrm{~h})$ pigs started to lie down in proximity to at least two others. There was no influence of genetics $\left(F_{1,105}=1.51 ; P=0.22\right)$ or housing conditions $\left(F_{1,105}=1.31 ; P=0.26\right)$ on the latency to lie down in a group. There was no $\mathrm{G} \times \mathrm{E}$ interaction (High B $102 \pm 12 \mathrm{~min}$; High E $115 \pm 13$; Low B $88 \pm 11$; Low E $101 \pm 12 ; F_{1,105}=$ $0.00 ; P=0.97)$, and no influence of sex or coping style.

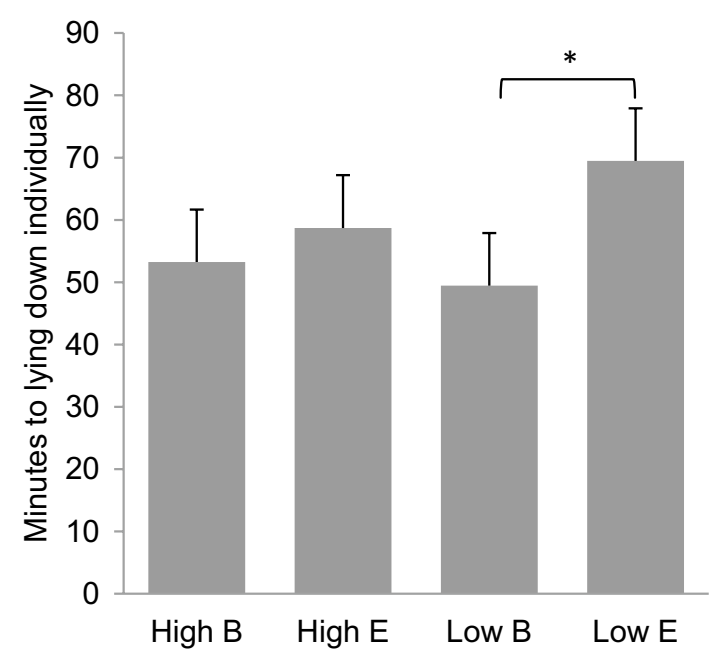

Fig. 3 Mean \pm SE latency (in minutes) to lie down individually after arrival in the experimental facilities (after being weaned and transported), by IGEg group (High/Low) and housing conditions (Barren, B / Enriched, E) 


\section{Discussion}

Pigs divergently selected for Indirect Genetic Effects for growth (IGEg) were housed in either conventional barren pens or straw enriched pens, creating a $\mathrm{G} \times \mathrm{E}$ set-up. Animals were studied around weaning, which is a stressful period for pigs which here coincided with separation from the dam, a $2.5 \mathrm{~h}$ journey by truck and a new environment with unfamiliar conspecifics and increased human handling. Low IGEg pigs had a shorter latency to start exploring or eating the feed and a greater weight gain over the first 4 days after weaning. Thus, in contrast with our hypothesis that Low IGEg might be more sensitive to stress, based on behavioural and physiological data at later age, they in fact responded seemingly more relaxed to this stressful situation.

In theory, animals selected for high IGEg during the grow-finisher phase would grow better when kept with group mates with similar social breeding value (as in our experiment), because they receive positive indirect genetic effects from the others in the group. The higher weight gain in Low IGEg, which seems contradictory, is however in line with previous work on IGEg in pigs (Rodenburg et al. 2010; Canario et al. 2012). The aforementioned studies found that pigs with a high breeding value for IGEg showed more aggression when grouped with unfamiliar conspecifics. Aggression at regrouping can result in a depression in growth performance (e.g. Tan et al. 1991), and this would be opposite to the expected positive effect on the growth of group members. At a few weeks after regrouping, however, pigs with a high IGEg showed less aggression than pigs with a low IGEg (Rodenburg et al. 2010; Canario et al. 2012). The former studies suggest that selection on high IGEg contributes to a better establishment of a stable dominance hierarchy, and thereby result in less aggression over the long term.

Previously reported data on the current study population showed that on the day after weaning the genetics did not influence the aggressive behaviour, but at 3 days after weaning High IGEg pigs indeed showed less aggression than Low IGEg (Camerlink et al. 2013). In addition, at later age High IGEg pigs showed less aggression upon reunion with group mates after $24 \mathrm{~h}$ separation for a regrouping test (Camerlink et al. 2013). Thus, the smaller weight gain of High IGEg pigs at weaning as well as the longer latency to explore or eat the feed may be related to High IGEg pigs investing more time into establishing social relationships upon first encounter.

Physiological factors that may differ due to selection on IGEg may, however, co-exits alongside any potential differences in the formation of dominance relationships. Based on additional behavioural and physiological data from the same animals in later life we here discuss the potential of alternative explanations.

Current commercial pig breeds have mostly been selected on fast growth and a high feed intake (Rauw et al. 1998). Highly productive animals will have a high, potentially unfulfilled, metabolic demand causing a strong motivation to eat (D'Eath et al. 2009). If this food motivation cannot be fulfilled then this may result in amongst others redirected oral manipulative behaviour and stereotypies (D'Eath et al. 2009; Ursinus et al. 2014c). When this oral manipulative biting is (re)directed towards conspecifics, the social interactions will become harmful, which may be reflected in IGEg (Turner 2011). Low IGEg pigs indeed showed higher levels of oral manipulative behaviours towards conspecifics and objects in the environment later in life (Camerlink et al. 2015). This may suggest that Low IGEg piglets might have a higher internally driven food motivation which would explain the short latency to start exploring or eating the feed despite the new environment and other likely stressful factors around weaning.

The above mentioned traits-oral manipulative behaviour, food motivation and growth - may be associated with the amino acid tryptophan. Tryptophan is an essential amino acid which plays a role in amongst others appetite, body protein deposition, growth performance and behaviour (Le Floc'h et al. 2011; Sève et al, 1991; Martınez-Trejo et al. 2009; Azmitia 2010). Tryptophan is a precursor for serotonin, and physiological data from the same animals as studied here showed variance in the functioning of the serotonergic system. Low IGEg pigs tended to have a higher serotonin platelet uptake velocity at 22 weeks of age compared to High IGEg pigs (untransformed LSmeans High IGEg = 41.6; Low $\mathrm{IGEg}=43.6 \mathrm{pmol} / 10^{9}$ platelets $/ \mathrm{min}, \mathrm{SE}=1.4 ; F_{1,332}=3,09$; $P=0.08$; unpublished results from the study described in Ursinus et al. 2014b). A higher platelet uptake velocity may imply that free serotonin in blood plasma is only shortly available to use and is quickly stored in blood platelets. The serotonergic system, which relates to the amount of available tryptophan, is known to be involved in stress-related disorders (e.g. Delorme et al. 2005; Coppen et al. 1976; Cleare 1997) and has found to be associated with oral manipulative behaviour in pigs (tail biting) (Ursinus et al. 2014b; Valros et al. 2015). Our findings suggest that high IGEg pigs may have a higher availability or lower utilization of the serotonin precursor tryptophan, which could explain the lower occurrence of oral manipulative behaviours.

The enteric microbiota has been suggested to play a role, through the gut-brain axis, in the intensity of oral manipulative behaviours in livestock (Brunberg et al. 2016). The host's genetics influence the composition and functioning of the gut microbiota (Hansen et al. 2010; pigs:; Zimmermann et al. 2016) and it is therefore worth considering the potential influence of genetic selection for IGEg on gut 
microbiota, as an alteration would affect both behaviour and growth (Cryan and Dinan 2012; Pascoe et al. 2017).

This study kept animals in two contrasting housing conditions to explore potential genotype-by-environment $(\mathrm{G} \times \mathrm{E})$ interactions. Half of the pigs were kept on a deep layer of straw, which is for pigs extremely appealing to explore and chew on (Fraser et al. 1991; Jensen et al. 2015) and may in some cases increase growth performance, although results are inconsistent about the effects on growth (reviewed by Millet et al. 2005). It thus created an additional novelty to explore, which may have made the animals more directed toward the environment rather than to explore the feed or to interact with conspecifics. In enriched pens, pigs were indeed more exploring, rooting, playing and chewing on straw in the first week after weaning (Camerlink et al. 2015). Hence, this may explain the longer latency to lie down in straw enriched pens, although it did not increase the latency to start eating. There were no $\mathrm{G} \times \mathrm{E}$ interactions for the behaviour and productivity at this age.

IGEg are currently being explored or taken into account by most of the major pig breeding companies. One aim of including IGE into breeding estimates is to gain a more accurate prediction of breeding values, which allows for an increase in response to selection. Theory predicts that a group of animals selected for high IGEg will grow better due to the positive effect on each other's growth (Bijma et al. 2007). In our experiment, parents of the pigs had been selected for their estimated IGE on growth during the entire fattening period. Our results suggest that the outcome of such selection may result in temporarily reduced growth, which could be due to aggression during the formation of dominance relationships. Stable dominance relationships can be beneficial in the long run and reduce the amount of aggression and unrest later in life (Turner et al. 2017). The effect of selection for IGEg on behaviour should therefore actually not be assessed based on a single time frame (Schneider et al. 2017). Rather, social interactions between group living species are dynamic rather than static, and the current explorations on combing the fields of social network analysis with IGE modelling are a promising avenue for further research.

\section{Conclusion}

Pigs divergently selected on Indirect Genetic Effects for growth (IGEg) showed differences in their behaviour and weight gain in the first 4 days after weaning. High IGEg animals were slower to explore the new feed and grew slower in the first 4 days after weaning than Low IGEg pigs. Although this seems opposite to the theory of selection for IGEg, the results are in line with previous studies which suggested that in newly formed groups high IGEg pigs may invest more energy in social interactions including aggression.

Acknowledgements This research was financially supported by The Netherlands Organisation for Scientific Research (NWO) and the Dutch Ministry of Economic Affairs. The experiment was part of the projects 'Seeking Sociable Swine' and 'Identification of biological characteristics that predict (mal)adaptation in pigs'. We are grateful to Topigs Norsvin for providing the pigs and pedigree data and to Naomi Duijvesteijn for performing the breeding values estimations and the selection of animals. We would like to thank Inonge Reimert, Monique Suer, Justine Jeanpierre, and Annemarie Vennix for their contribution to the data collection.

Author contributions IC, WWU, PB, and JEB designed the experiment. IC, WWU, and ACB collected the data. IC and WWU wrote and prepared the manuscript for submission. JEB and PB reviewed the manuscript.

Funding This study was funded by The Netherlands Organisation for Scientific Research (NWO) and the Dutch Ministry of Economic Affairs (grant number 827.09.010 and KB8-4432666700).

\section{Compliance with ethical standards}

Conflict of interest The authors Irene Camerlink, Winanda W. Ursinus, Andrea C. Bartels, Piter Bijma, J. Elizabeth Bolhuis declare that they have no competing interests.

Human and Animal Rights All applicable international, national, and/ or institutional guidelines for the care and use of animals were followed. The study was carried out in strict accordance with the recommendations in the European Guidelines for accommodation and care of animals. The protocol was approved by the Institutional Animal Care and Use Committee of Wageningen University (Protocol Number: 2010055f).

Informed consent Informed consent was obtained from all individual participants included in the study.

Open Access This article is distributed under the terms of the Creative Commons Attribution 4.0 International License (http://creativeco mmons.org/licenses/by/4.0/), which permits unrestricted use, distribution, and reproduction in any medium, provided you give appropriate credit to the original author(s) and the source, provide a link to the Creative Commons license, and indicate if changes were made.

\section{References}

Azmitia EC (2010) Evolution of serotonin: sunlight to suicide. In: Müller CR, Jacobs B (eds) Handbook of the behavioural neurobiology of serotonin. Academic Press, London, pp 3-22

Bailey NW, Marie-Orleach L, Moore AJ (2017) Indirect genetic effects in behavioral ecology: does behavior play a special role in evolution? Behav Ecol 29(1):1-11

Bergsma R, Mathur PK, Kanis E, Verstegen MWA, Knol EF, Van Arendonk JAM (2013) Genetic correlations between lactation performance and growing-finishing traits in pigs. J Anim Sci 91(8):3601-3611 
Bijma P (2014) The quantitative genetics of indirect genetic effects: a selective review of modelling issues. Heredity 112(1):61

Bijma P, Wade MJ (2008) The joint effects of kin, multilevel selection and indirect genetic effects on response to genetic selection. J Evol Biol 21(5):1175-1188

Bijma P, Muir WM, Ellen ED, Wolf JB, Van Arendonk JA (2007) Multilevel selection 2: estimating the genetic parameters determining inheritance and response to selection. Genetics 175(1):289-299

Bolhuis JE, Parmentier HK, Schouten WGP, Schrama JW, Wiegant VM (2003) Effects of housing and individual coping characteristics on immune responses of pigs. Physiol Behav 79:289-296

Bolhuis JE, Schouten WGP, De Leeuwa JA, Schrama JW, Wiegant VM (2004) Research report Individual coping characteristics, rearing conditions and behavioural flexibility in pigs. Behav Brain Res 152:351-360

Brunberg EI, Rodenburg TB, Rydhmer L, Kjaer JB, Jensen P, Keeling LJ (2016) Omnivores going astray: a review and new synthesis of abnormal behavior in pigs and laying hens. Front Vet Sci 3:57

Camerlink I (2014). Sociable swine: indirect genetic effects on growth rate and their effect on behaviour and production of pigs in different environments. PhD Dissertation. Wageningen University Press, Netherlands

Camerlink I, Turner SP, Bijma P, Bolhuis JE (2013). Indirect genetic effects and housing conditions in relation to aggressive behaviour in pigs. PLoS ONE, 8(6), e65136

Camerlink I, Bolhuis JE, Duijvesteijn N, Van Arendonk JAM, Bijma $P$ (2014) Growth performance and carcass traits in pigs selected for indirect genetic effects on growth rate in two environments. J Anim Sci 92(6):2612-2619

Camerlink I, Ursinus WW, Bijma P, Kemp B, Bolhuis JE (2015) Indirect genetic effects for growth rate in domestic pigs alter aggressive and manipulative biting behaviour. Behav Genet 45(1):117-126

Campbell JM, Crenshaw JD, Javier P (2013) The biological stress of early weaned piglets. J Anim Sci Biotechnol 4:19

Canario L, Turner SP, Roehe R, Lundeheim N, D'Eath RB, Lawrence AB , ... Rydhmer L (2012) Genetic associations between behavioral traits and direct-social effects of growth rate in pigs. J Anim Sci 90(13):4706-4715

Cleare AJ (1997) Reduced whole blood serotonin in major depression. Depress Anxiety 5:108-111

Coppen A, Turner P, Rowsell AR, Padgham C (1976) 5 Hydroxytryptamine (5 HT) in the whole blood of patients with depressive illness. Postgrad Med J 52:156-158. 59

Costa e Silva J, Kerr RJ (2013) Accounting for competition in genetic analysis, with particular emphasis on forest genetic trials. Tree Genet Genomes 9(1):1-17

Cryan JF, Dinan TG (2012) Mind-altering microorganisms: the impact of the gut microbiota on brain and behaviour. Nat Rev Neurosci 13(10):701

D’Eath RB, Tolkamp BJ, Kyriazakis I, Lawrence AB (2009) 'Freedom from hunger' and preventing obesity: the animal welfare implications of reducing food quantity or quality. Anim Behav 77(2):275-288

Delorme R, Betancur C, Callebert J, Chabane N, Laplanche JL, Mouren-Simeoni MC, Launay JM, Leboyer M (2005) Platelet serotonergic markers as endophenotypes for obsessive-compulsive disorder. Neuropsychopharmacology 30:1539-1547. 58

Duijvesteijn N, Knol EF, Bijma P (2012) Direct and associative effects for androstenone and genetic correlations with backfat and growth in entire male pigs. J Anim Sci 90(8):2465-2475

Ellen ED, Rodenburg TB, Albers GA, Bolhuis JE, Camerlink I, Duijvesteijn N, Knol EF, Muir WM, Peeters K, Reimert I, Sell-Kubiak $E$ (2014). The prospects of selection for social genetic effects to improve welfare and productivity in livestock. Front Genet 5:377
Fraser D, Phillips PA, Thompson BK, Tennessen T (1991) Effect of straw on the behaviour of growing pigs. Appl Anim Behav Sci 30(3):307-318

Griffing B (1967) Selection in reference to biological groups I. Individual and group selection applied to populations of unordered groups. Aust J Biol Sci 20(1):127-140

Hansen J, Gulati A, Sartor RB (2010) The role of mucosal immunity and host genetics in defining intestinal commensal bacteria. Curr Opin Gastroenterol 26(6):564

Hessing MJ, Hagels $\varnothing$ AM, Van Beek JAM, Wiepkema RP, Schouten WGP, Krukow R (1993) Individual behavioural characteristics in pigs. Appl Anim Behav Sci 37:285-295

Iversen MW, Bolhuis JE, Camerlink I, Ursinus WW, Reimert I, Duijvesteijn N (2017) Heritability of the backtest response in piglets and its genetic correlations with production traits. Animal 11(4):556-563

Jensen MB, Herskin MS, Forkman B, Pedersen LJ (2015) Effect of increasing amounts of straw on pigs' explorative behaviour. Appl Anim Behav Sci 171:58-63

Le Floc'h N, Otten W, Merlot E (2011) Tryptophan metabolism, from nutrition to potential therapeutic applications. Amino Acids 41:1195-1205

Lipschutz-Powell D, Woolliams JA, Bijma P, Doeschl-Wilson AB (2012). Indirect genetic effects and the spread of infectious disease: are we capturing the full heritable variation underlying disease prevalence? PLoS ONE, 7(6):e39551

Martínez-Miró S, Tecles F, Ramón M, Escribano D, Hernández F, Madrid J, Orengo J, Martínez-Subiela S, Manteca X, Cerón JJ (2016) Causes, consequences and biomarkers of stress in swine: an update. BMC Vet Res 12:171

Martınez-Trejo G, Ortega-Cerrilla ME, Rodarte-Covarrubias LF, Herrera-Haro JG, Figueroa-Velasco JL, Galindo-Maldonado F, Sanchez-Martınez O, Lara-Bueno A (2009) Aggressiveness and productive performance of piglets supplemented with tryptophan. J Vet Adv 8:608ஷ̀611

Melotti L, Oostindjer M, Bolhuis JE, Held S, Mendl M (2011) Coping personality type and environmental enrichment affect aggression at weaning in pigs. Appl Anim Behav Sci 133(3):144-153

Millet S, Moons CP, Van Oeckel MJ, Janssens GP (2005) Welfare, performance and meat quality of fattening pigs in alternative housing and management systems: a review. J Sci Food Agric 85(5):709-719

Moore AJ, Brodie ED, Wolf JB (1997) Interacting phenotypes and the evolutionary process: I. Direct and indirect genetic effects of social interactions. Evolution 51(5):1352-1362

Muir WM (2005) Incorporation of competitive effects in forest tree or animal breeding programs. Genetics 170(3):1247-1259

Oostindjer M, Bolhuis JE, Simon K, van den Brand H, Kemp B, (2011a). Perinatal flavour learning and adaptation to being weaned: all the pig needs is smell. PLoS ONE 6, e25318

Oostindjer M, Muñoz JM, Van den Brand H, Kemp B, Bolhuis JE (2011b) Maternal presence and environmental enrichment affect food neophobia of piglets. Biol Let 7(1):19-22

Oostindjer M, van den Brand H, Kemp B, Bolhuis JE (2011c) Effects of environmental enrichment and loose housing of lactating sows on piglet behaviour before and after weaning. Appl Anim Behav Sci 134(1):31-41

Pascoe EL, Hauffe HC, Marchesi JR, Perkins SE (2017) Network analysis of gut microbiota literature: an overview of the research landscape in non-human animal studies. ISME J 11(12):2644

Peeters K, Eppink TT, Ellen ED, Visscher J, Bijma P (2012) Indirect genetic effects for survival in domestic chickens (Gallus gallus) are magnified in crossbred genotypes and show a parent-of-origin effect. Genetics 192(2):705-713

Potts BM, Bijma P, Kerr RJ, Pilbeam DJ (2013) Genetic control of interactions among individuals: contrasting outcomes of indirect 
genetic effects arising from neighbour disease infection and competition in a forest tree. New Phytol 197(2):631-641

Rauw WM, Kanis E, Noordhuizen-Stassen EN, Grommers FJ (1998) Undesirable side effects of selection for high production efficiency in farm animals: a review. Livest Prod Sci 56(1):15-33

Reimert I, Rodenburg TB, Ursinus WW, Duijvesteijn N, Camerlink I, Kemp B, Bolhuis JE (2013) Backtest and novelty behavior of female and castrated male piglets, with diverging social breeding values for growth. J Anim Sci 91(10):4589-4597

Reimert I, Rodenburg TB, Ursinus WW, Kemp B, Bolhuis JE (2014a) Responses to novel situations of female and castrated male pigs with divergent social breeding values and different backtest classifications in barren and straw-enriched housing. Appl Anim Behav Sci 151:24-35

Reimert I, Rodenburg TB, Ursinus WW, Kemp B, Bolhuis JE (2014b). Selection based on indirect genetic effects for growth, environmental enrichment and coping style affect the immune status of pigs. PLoS ONE 9(10):e108700

Rodenburg TB, Bijma P, Ellen ED, Bergsma R, De Vries S, Bolhuis JE et al (2010) Breeding amiable animals? Improving farm animal welfare by including social effects in breeding programmes. Anim Welf 19(2):77-82

Schneider J, Atallah J, Levine JD (2017) Social structure and indirect genetic effects: genetics of social behaviour. Biol Rev 92(2):1027-1038

Sève B, Meunier-Salaün MC, Monnier M, Colléaux Y, Henry Y (1991) Impact of dietary tryptophan and behavioral type on growth performance and plasma amino acids of young pigs. J Anim Sci 69(9):3679-3688

Tan SSL, Shackleton DM, Beames RM (1991) The effect of mixing unfamiliar individuals on the growth and production of finishing pigs. Anim Sci 52(1):201-206

Turner SP (2011) Breeding against harmful social behaviours in pigs and chickens: state of the art and the way forward. Appl Anim Behav Sci 134(1):1-9
Turner SP, Nevison IM, Desire S, Camerlink I, Roehe R, Ison SH, ... D'Eath RB (2017) Aggressive behaviour at regrouping is a poor predictor of chronic aggression in stable social groups. Appl Anim Behav Sci 191:98-106

Ursinus WW, Bolhuis JE, Zonderland JJ, Rodenburg TB, De Souza AS, Koopmanschap RE, Kemp B, Korte-Bouws GA, Korte SM, Van Reenen CG (2013). Relations between peripheral and brain serotonin measures and behavioural responses in a novelty test in pigs. Physiol Behav 118:88-96

Ursinus WW, Van Reenen CG, Kemp B, Bolhuis JE (2014a) Tail biting behaviour and tail damage in pigs and the relationship with general behaviour: predicting the inevitable? Appl Anim Behav Sci 156:22-36

Ursinus WW, Van Reenen CG, Reimert I, Bolhuis JE (2014b). Tail biting in pigs: blood serotonin and fearfulness as pieces of the puzzle? PLoS ONE 9:e107040

Ursinus WW, Wijnen HJ, Bartels AC, Dijvesteijn N, van Reenen CG, Bolhuis JE (2014c) Damaging biting behaviors in intensively kept rearing gilts: the effect of jute sacks and relations with production characteristics. J Anim Sci 92:5193-5202

Valros A, Palander P, Heinonen M, Munsterhjelm C, Brunberg E, Keeling L, Piepponen P (2015) Evidence for a link between tail biting and central monoamine metabolism in pigs (Sus scrofa domestica). Physiol Behav 143:151-157

Wolf JB, Brodie ED, III, Cheverud JM, Moore AJ, Wade MJ (1998) Evolutionary consequences of indirect genetic effects. Trends Ecol Evol 13(2):64-69

Zimmermann JA, Fusari ML, Rossler E, Blajman JE, Romero-Scharpen A, Astesana DM et al (2016) Effects of probiotics in swines growth performance: a meta-analysis of randomised controlled trials. Anim Feed Sci Technol 219:280-293 